\title{
Upregulation of myocellular DGAT1 augments triglyceride synthesis in skeletal muscle and protects against fat-induced insulin resistance
}

\author{
Li Liu, ${ }^{1}$ Yiying Zhang, ${ }^{2}$ Nancy Chen, ${ }^{1}$ Xiaojing Shi, ${ }^{1}$ Bonny Tsang, ${ }^{1}$ and Yi-Hao Yu ${ }^{1}$ \\ ${ }^{1}$ Department of Medicine and 2Department of Pediatrics, Columbia University, New York, New York, USA.
}

\begin{abstract}
Increased fat deposition in skeletal muscle is associated with insulin resistance. However, exercise increases both intramyocellular fat stores and insulin sensitivity, a phenomenon referred to as "the athlete's paradox". In this study, we provide evidence that augmenting triglyceride synthesis in skeletal muscle is intrinsically connected with increased insulin sensitivity. Exercise increased diacylglycerol (DAG) acyltransferase (DGAT) activity in skeletal muscle. Channeling fatty acid substrates into TG resulted in decreased DAG and ceramide levels. Transgenic overexpression of DGAT1 in mouse skeletal muscle replicated these findings and protected mice against high-fat diet-induced insulin resistance. Moreover, in isolated muscle, DGAT1 deficiency exacerbated insulin resistance caused by fatty acids, whereas DGAT1 overexpression mitigated the detrimental effect of fatty acids. The heightened insulin sensitivity in the transgenic mice was associated with attenuated fat-induced activation of DAG-responsive PKCs and the stress mediator JNK1. Consistent with these changes, serine phosphorylation of insulin receptor substrate 1 was reduced, and Akt activation and glucose 4 membrane translocation were increased. In conclusion, upregulation of DGAT1 in skeletal muscle is sufficient to recreate the athlete's paradox and illustrates a mechanism of exercise-induced enhancement of muscle insulin sensitivity. Thus, increasing muscle DGAT activity may offer a new approach to prevent and treat insulin resistance and type 2 diabetes mellitus.
\end{abstract}

\section{Introduction}

In obesity, total-body energy excess may exceed the storage capacity of adipose tissue. As a result, "ectopic" triglyceride (TG) deposition occurs in tissues not specialized for fat storage and causes lipotoxicity $(1,2)$. Skeletal muscle is the major peripheral tissue in which insulin-responsive glucose uptake and utilization take place. Increased fat deposition in muscle is associated with insulin resistance in both humans and rodents (3-10), whereas reduction of elevated intracellular TG content in muscle leads to improved insulin sensitivity (11-14). However, whether intramyocellular TG (IMTG) is a cause of or a marker for the associated muscle insulin resistance remains unresolved, primarily due to the fact that increased IMTG in fatty acid (FA) overload states is accompanied by increased intramyocellular levels of other FA derivatives that also have the potential for lipotoxicity (15-20).

In contrast to the positive correlation between elevated IMTG and insulin resistance in obesity and type 2 diabetes, such a relationship does not hold true in trained athletes. Exercise also increases muscle fat stores (21-24), but improves insulin sensitivity $(22,25-32)$, a finding that is referred to as "the athlete's paradox" $(22,33)$. The athlete's paradox is not restricted to athletes, however. In fact, short-term exercise or a single session of exercise also induces accumulation of IMTG in untrained individuals (23, 24). The mechanism by which insulin sensitivity is preserved or increased under these conditions is unknown.

Nonstandard abbreviations used: AUC, area under the curve; DAG, diacylglycerol; DGAT, DAG acyltransferase; FA, fatty acid; GLUT4, glucose transporter 4; GTT, glucose tolerance tests; HFD, high-fat diet; IMTG, intramyocellular TG; IRS-1, insulin receptor substrate 1 ; ITT, insulin tolerance tests; MCK, muscle creatine kinase; NC, normal chow; RBP4, retinol binding protein 4; TG, triglyceride.

Conflict of interest: The authors have declared that no conflict of interest exists. Citation for this article: J. Clin. Invest. 117:1679-1689 (2007). doi:10.1172/JCI30565
Exercise increases mitochondrial FA $\beta$-oxidation, which is believed to play an important role in maintaining muscle insulin sensitivity $(22,30-32)$. Long-term exercise training may also lead to myofiber remodeling, with increased mitochondrion biogenesis and functions and/or expansion of type I oxidative myofibers (34). Type I oxidative myofibers are slow-twitch fibers that have a higher TG content and lower glycogen storage. Despite their oxidative (as opposed to glycolytic) nature, type I myofibers typically have higher glucose transporter 4 (GLUT4) content and are more responsive to insulin-mediated glucose disposal (35-39). Despite these findings, the nature of the relationship between the increased IMTG and the improve muscle insulin sensitivity in exercise remains unclear.

Diacylglycerol (DAG) acyltransferase (DGAT) catalyzes the last step of the glycerol phosphate pathway of TG synthesis in mammalian cells. DGAT produces TG from its 2 substrates, DAG and fatty acyl-CoA $(40,41)$. In this capacity, DGAT promotes TG storage but may also decrease FA substrates (42). Two DGAT genes have been cloned; both are expressed in many types of tissues, including adipose tissue, liver, intestines, and skeletal muscles, although differences in relative abundance are also evident (43-45). The 2 DGAT genes bear no sequence homology to each other. DGAT1 is a member of the mammalian acyl-CoA cholesterol acyltransferase (ACAT) family $(43,44)$, whereas DGAT2 belongs to a new family and was cloned by a homolog search based on the sequence of the fungal DGAT2 (46). Although both enzymes catalyze the same reaction in TG synthesis, they are functionally distinguished by their differences in regulation (47), phenotypic consequences when rendered deficient $(48,49)$, and additional functions (DGAT1 also esterifies alcohol and retinol; refs. 50, 51).

Since exercise induces the expression of DGAT1 in muscle cells (52), we investigated whether DGAT1-mediated augmentation of TG synthesis in muscle is causally associated with exercise-induced 

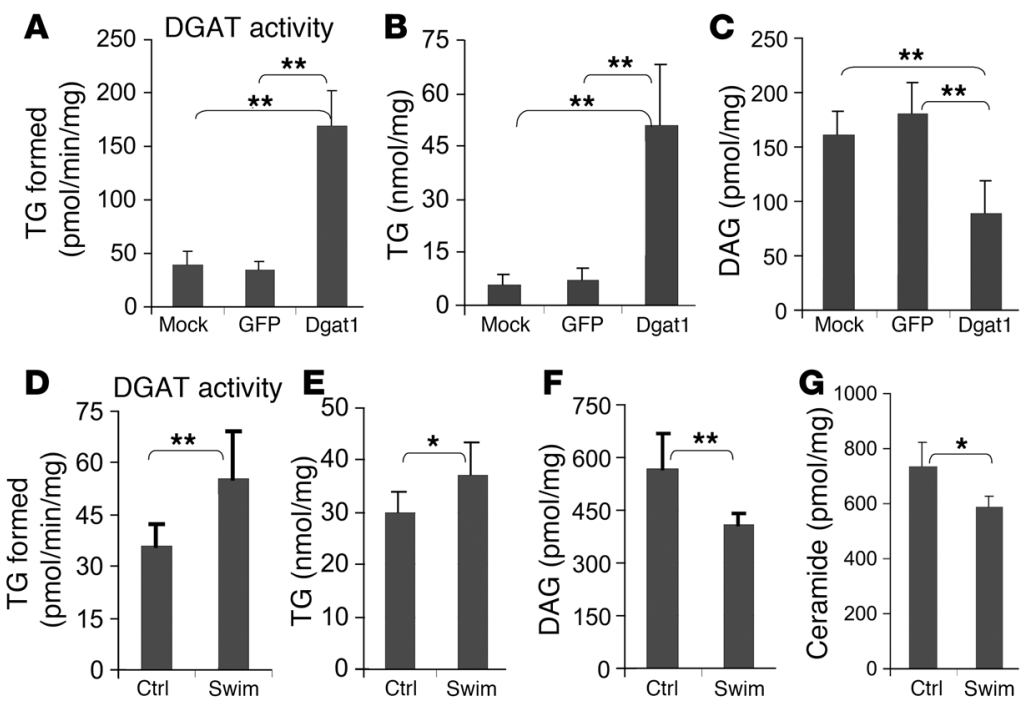

\section{Figure 1}

Upregulation of myocellular DGAT increases intracellular TG levels and decreases intracellular DAG levels. (A-C) Overexpression of DGAT1 via recombinant adenovirus (Dgat1) in differentiated C2C12 myocytes, showing DGAT activity levels (A), TG content (B), and DAG level (C) compared with mock-transduced cells or cells transduced with a control recombinant adenovirus expressing GFP. (D-G) Exercise-induced DGAT activation (D) and changes in myocellular TG $(\mathbf{E})$, DAG (F), and ceramide (G) levels in isolated soleus muscles from 3-month-old male mice after 1 week swimming training (Swim; $n=6$ ) as compared with age-, gender-, and diet-matched sedentary mice (Ctrl; $n=4)$. ${ }^{*} P<0.05 ;{ }^{* *} P<0.01$. improvement of muscle insulin sensitivity. We hypothesized that upregulation of DGAT1 would facilitate the removal of lipotoxic FA derivatives by channeling FA substrates into storage in the form of TG. In the present study, we provide evidence that muscle insulin sensitivity is intrinsically connected with the myocellular TG synthesis capacity and that augmenting myocellular TG synthesis in transgenic mice with overexpression of DGAT1 in skeletal muscle is sufficient to improve muscle insulin sensitivity.

\section{Results}

Augmentation of myocellular TG synthesis is associated with increased IMTG and decreased DAG and ceramide. To determine whether overexpression of DGAT1 in myocytes can cause not only an increase in the enzyme product, but also a decrease in the enzyme substrates, we overexpressed DGAT1 in differentiated C2C12 myocytes via recombinant adenoviruses. Delivery of the DGAT1 adenovirus to C2C12 myocytes caused a 3-fold increase in DGAT activity (Figure 1A), a 6 -fold increase in IMTG (Figure 1B), and a 50\% decrease in DAG levels (Figure 1C) compared with the mock transduced cells or cells transduced with a GFP adenovirus.

We then investigated whether an increase of IMTG by exercise was also associated with decreased lipotoxic FA derivatives. We compared 2 groups of age-matched and weight-matched male mice, an exercise group ( 6 swimming sessions per day for 1 week) and a control group (no swimming sessions). The

\section{Figure 2}

Transgenic mice with DGAT1 overexpression in skeletal muscle. (A) The Dgat1 transgene contains (from the $5^{\prime}$ end to the $3^{\prime}$ end) the 3.3-kb MCK promoter, a human Dgat1 cDNA containing its own initiation and termination codons, and the genomic sequence of human growth hormone (hGH) containing the last 3 exons and 2 introns as indicated. (B) Tissue distribution of the Dgat1 transgene mRNA levels measured by reverse transcription and PCR amplification. $\mathrm{S}$. intestine, small intestine; gastro., gastrocnemius. (C) RT-PCR quantification of total DGAT1 and DGAT2 mRNA levels in soleus muscles of WT and transgenic mice ( $n=5$ in each group). (D) Total DGAT activity levels in membrane fractions of soleus muscles from WT and transgenic mice ( $n=5$ in each group). ${ }^{* \star} P<0.01$. exercise-trained mice had a $70 \%$ increase in muscle DGAT activity (Figure 1D), which was associated with approximately $150 \%$ increases in DGAT1 mRNA levels (Supplemental Figure 1; supplemental material available online with this article; doi:10.1172/ JCI30565DS1). IMTG levels were 24\% increased (Figure 1E), but DAG and ceramide levels were $28 \%$ and $20 \%$ decreased in soleus muscles of exercise-trained mice (Figure 1, F and G, respectively), compared with those of the control mice. Although differing in magnitudes, these results mirror the findings in DGAT1-overexpressing myocytes (Figure 1, A-C) and suggest that exerciseinduced DGAT activation may be involved in the manifestation of the athlete's paradox.

Overexpression of DGAT1 in mouse skeletal muscle recapitulates the athlete's paradox and protects against bigh-fat diet-induced insulin resistance. We created transgenic mice with DGAT1 overexpression in skeletal muscle under the control of the muscle creatine kinase (MCK) promoter (Figure 2A). RT-PCR showed that expression of
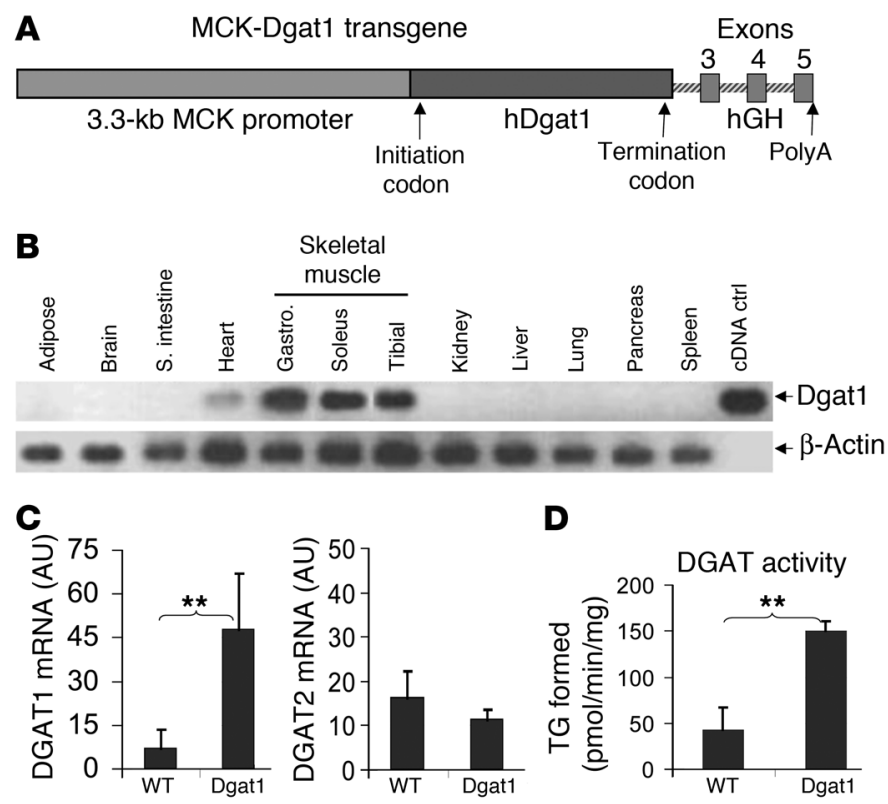

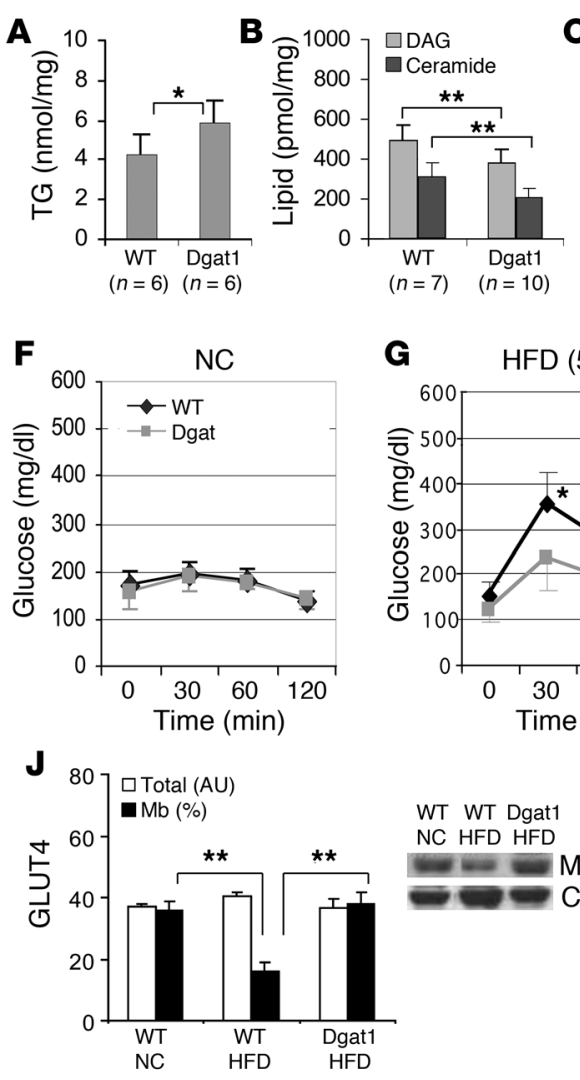
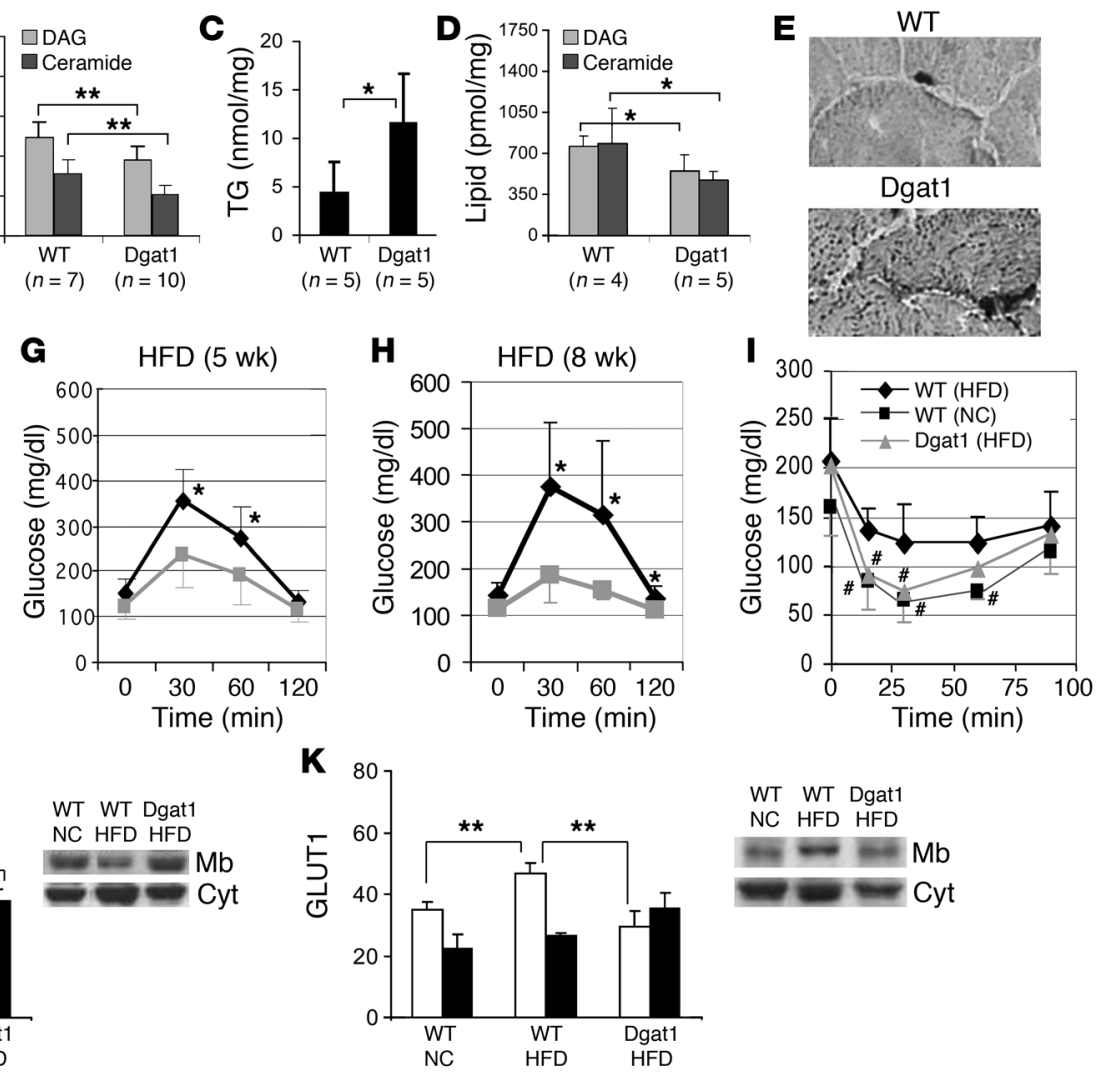

\section{K}

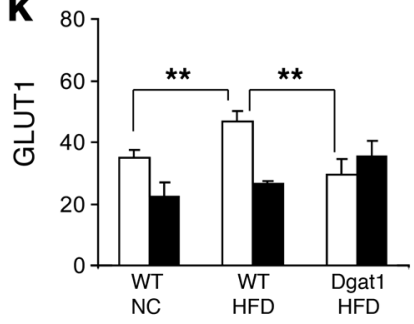

WT WT Dgat1 NC HFD HFD

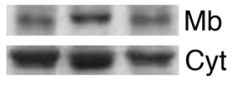

Figure 3

Myocellular DGAT1 overexpression reproduces the athlete's paradox and protects against FA-induced insulin resistance. (A and B) Muscle TG (A) and DAG and ceramide (B) contents in soleus muscles isolated from WT and transgenic mice on NC diet. (C and D) Muscle TG (C) and DAG and ceramide (D) contents in soleus muscles isolated from WT and transgenic mice after 8 weeks of HFD treatment. (E) Representative cross-sections of soleus muscles from HFD-fed WT and MCK-Dgat1 transgenic mice, stained for neutral lipids (primarily TG) with oil red O. (F-H) GTT in age- and diet-matched male WT $(n=9)$ and transgenic $(n=5)$ mice on NC diet (F), HFD for 5 weeks (G), or HFD for 8 weeks (H). (I) ITT in age-matched male WT and transgenic mice on NC diet or HFD for 8 weeks. ${ }^{P}<0.05$ compared with HFD-fed WT mice. (J) Quantification of total and percentage of membrane-bound GLUT4 in soleus muscles isolated from WT mice on NC diet, WT mice on HFD, and transgenic mice on HFD. The quantification is based on Western blot analysis of the myocellular membrane-bound (Mb) and cytosol (Cyt) fractions of GLUT4 ( $n=4$ in each group); a typical Western blot is presented as an inset. (K) Quantification of total and percentage of membrane-bound GLUT1 from the muscle specimens described in $\mathrm{J}$. ${ }^{\star} P<0.05 ;{ }^{*} P<0.01$.

the transgene was primarily restricted to skeletal muscle, with high levels of expression in various groups of skeletal muscles including gastrocnemius, soleus, and posterior tibial muscles, but a very low expression level in the heart (Figure 2B and Supplemental Figure 2A). In isolated soleus muscle, total DGAT1 mRNA was increased by 5 -fold, and DGAT2 mRNA expression was unchanged (Figure 2C); total DGAT activity was increased by approximately 3 -fold (Figure 2D). A 2- to 4-fold increase in total DGAT activity was also consistently observed in other skeletal muscle groups of the transgenic mice, compared with respective muscles of WT mice (Supplemental Figure 2B).

We sought to determine whether the transgenic mice were able to reproduce the athlete's paradox. In normal chow-fed (NC-fed) mice, IMTG levels in soleus muscle were 37\% increased in MCKDgat 1 mice compared with WT mice (Figure 3A), while DAG and ceramide levels were $23 \%$ and $35 \%$ decreased, respectively (Figure 3B). After 8 weeks of high-fat diet (HFD) feeding, IMTG levels were markedly increased in transgenic mice but were only modestly increased in WT mice (in Figure 3, compare C with A). IMTG levels were approximately $120 \%$ higher in HFD-fed transgenic mice than in HFD-fed WT mice (Figure 3C). Increased muscle TG levels in transgenic mice appeared to be distributed intracellularly as distinct lipid droplets stained with oil red O (Figure 3E). Similar to the differences in lipid levels between WT and MCK-Dgat1 mice on NC diet, muscle DAG and ceramide levels in HFD-fed transgenic mice were also approximately $30 \%$ and $25 \%$ lower, respectively, than in WT mice (Figure 3D). Despite the increased IMTG levels, there was no evidence of insulin resistance in the transgenic mice fed the NC diet; their fasting glucose and insulin levels were indistinguishable from those of the WT mice (Table 1). Glucose tolerance tests (GTT) showed no difference between the transgenic and WT mice on NC diet (Figure 3F). Five and eight weeks after HFD feeding, progressive glucose intolerance became evident in WT mice (Figure 3, G and H, respectively). However HFD-fed MCKDgat 1 mice maintained normal glucose tolerance (Figure 3, G and $\mathrm{H})$. Similarly, insulin tolerance tests (ITT) showed marked insulin resistance in HFD-fed WT mice but not in HFD-fed Dgat 1 mice (Figure 3I). Plasma levels of adiponectin, IL-6, TNF- $\alpha$, resistin, and retinol binding protein 4 (RBP4) were not significantly different between HFD-fed WT and MCK-Dgat1 mice, except for a trend 


\section{Table 1}

The energetics and fasting plasma glucose and insulin levels in 3-month-old male and female WT and MCK-Dgat1 transgenic mice fed NC diet or 8 weeks of HFD

\begin{tabular}{|c|c|c|c|c|c|c|}
\hline & Sex & $\begin{array}{l}\text { Food intake } \\
(\mathrm{g} / \mathrm{g} / \mathrm{d})\end{array}$ & $\begin{array}{l}\text { Body weight } \\
\text { (g) }\end{array}$ & $\begin{array}{l}\text { \% Adipose } \\
\text { tissue }\end{array}$ & $\begin{array}{l}\text { Fasting plasma } \\
\text { glucose (mg/dl) }\end{array}$ & $\begin{array}{l}\text { Fasting plasma } \\
\text { insulin (ng/ml) }\end{array}$ \\
\hline \multicolumn{7}{|l|}{ NC-fed } \\
\hline WT $(n=6)$ & Male & $0.192 \pm 0.012$ & $32.1 \pm 2.8$ & $25.1 \pm 3.7$ & $95 \pm 9$ & $0.62 \pm 0.41$ \\
\hline MCK-Dgat1 $(n=5)$ & Male & $\begin{array}{c}0.191 \pm 0.009 \\
P=0.84\end{array}$ & $\begin{array}{c}30.6 \pm 2.1 \\
P=0.14\end{array}$ & $\begin{array}{c}23.9 \pm 4.1 \\
P=0.21\end{array}$ & $\begin{array}{r}93 \pm 14 \\
P=0.76\end{array}$ & $\begin{array}{c}0.58 \pm 0.33 \\
P=0.81\end{array}$ \\
\hline WT $(n=4)$ & Female & $0.186 \pm 0.008$ & $26.0 \pm 1.5$ & $20.6 \pm 5.6$ & $87 \pm 13$ & $0.30 \pm 0.28$ \\
\hline MCK-Dgat1 $(n=5)$ & Female & $\begin{array}{c}0.187 \pm 0.007 \\
P=0.94\end{array}$ & $\begin{array}{c}24.8 \pm 2.9 \\
P=0.09\end{array}$ & $\begin{array}{c}19.8 \pm 8.0 \\
P=0.39\end{array}$ & $\begin{array}{r}84 \pm 15 \\
P=0.62\end{array}$ & $\begin{array}{c}0.32 \pm 0.18 \\
P=0.83\end{array}$ \\
\hline \multicolumn{7}{|l|}{ HFD-fed } \\
\hline WT $(n=7)$ & Male & $\mathrm{nm}$ & $37.5 \pm 4.3$ & $33.7 \pm 0.07$ & $138 \pm 10$ & $2.73 \pm 0.96$ \\
\hline MCK-Dgat1 $(n=8)$ & Male & $\begin{array}{c}\mathrm{nm} \\
P=0.24\end{array}$ & $\begin{array}{c}34.7 \pm 4.6 \\
P=0.25\end{array}$ & $\begin{array}{c}28.4 \pm 0.10 \\
P=0.078\end{array}$ & $\begin{array}{l}119 \pm 22 \\
P=0.056\end{array}$ & $1.54 \pm 0.60$ \\
\hline
\end{tabular}

Data are expressed as mean \pm SD. $\mathrm{nm}$, not measured.

toward a decrease in IL-6 levels in transgenic mice (a mean of 0.22 $\mathrm{ng} / \mathrm{ml}$ versus $0.88 \mathrm{ng} / \mathrm{ml}, P=0.14$ ) (Table 2 ). Muscle specimens isolated after 8 weeks of HFD feeding showed decreased membrane translocation of GLUT4 in WT mice. This did not occur in Dgat1 mice fed HFD (Figure 3J). Membrane GLUT1 was increased in HFD-fed WT mice (Figure 3K), likely reflecting a compensatory response to decreased insulin sensitivity and GLUT4-mediated glucose transport. This compensatory response was largely absent in HFD-fed Dgat 1 mice, providing supporting evidence that insulin sensitivity was not compromised in the transgenic mice.

To verify that myocellular DGAT1 overexpression was responsible for preserving insulin sensitivity against lipid overload, muscles lacking DGAT1 were examined. Soleus muscles were isolated from DGAT1-knockout mice (48), MCK-Dgat1 transgenic mice, and WT mice (all in C57BL/6 genetic background) for comparison. After extensive washes to remove circulating factors, the muscles were further incubated for 2 hours in Krebs-Henseleit bicarbonate buffer in the absence or presence of albumin-conjugated FA ( $0.375 \mathrm{mM}$ palmitate plus $0.375 \mathrm{mM}$ oleate) to induce insulin resistance. Following the incubation, each set of muscles was assayed for 2-deoxyglucose uptake in the basal and insulinstimulated states. While basal 2-deoxyglucose uptakes were similar among all 3 groups with or without FA pretreatment (Figure 4, A and C), insulin-stimulated uptakes were dependent on the DGAT1 genotypes. Without FA pretreatment, insulin increased 2-deoxyglucose uptakes in the WT, transgenic, and knockout muscles by $123 \%(P<0.01), 180 \%(P<0.01)$, and 60\% $(P<0.05)$, respectively, above their basal uptake levels (Figure 4 , $A$ and $B)$. Two hours of preincubation with $0.75 \mathrm{mM}$ FA led to reduced insulinstimulated 2-deoxyglucose uptakes in all groups (Figure 4, C and D). However, the degree of reduction appears also to be genotype dependent: an approximately 5 -fold reduction in the WT muscle (from $123 \%$ to $26 \%$ ) and a complete elimination of insulin-stimulated uptake in the Dgat $1^{-/}$muscle (from $60 \%$ to $0 \%$ ) were observed, but there was less reduction in the transgenic muscle ( $\sim 3$-fold, from
$180 \%$ to $61 \%$ ) (Figure 4, A-D). Quantification of lipid contents in FA-pretreated muscles showed that TG levels were 58\% increased $(P<0.05)$ in the transgenic muscle and $46 \%$ decreased $(P<0.05)$ in the knockout muscle, as compared with the WT muscle (Figure 4E). In contrast, DAG and ceramide levels were 20\% $(P=0.075)$ and $39 \%(P<0.01)$ decreased, respectively, in the transgenic muscle, while they were $33 \%(P<0.05)$ and $26 \%(P=0.059)$ increased, respectively, in the knockout muscle (Figure 4, F and G).

$D G A T 1$ overexpression attenuates DAG-responsive $P K C$ and results in insulin sensitization. To determine the mechanisms by which the DGAT1 transgene mediates the protection against diet-induced insulin resistance, we examined the relationship between insulin sensitivity and intramyocellular levels of lipids in MCK-Dgat1 mice. Simple regression analysis showed a trend of inverse correlation between IMTG and the area under the curve (AUC) values of GTT (adjusted $R^{2}=0.126, P=0.084$; Figure 5A). Moreover, it revealed strong positive correlations between DAG and AUC levels (adjusted $R^{2}=0.515, P<0.001$; Figure 5B) and between ceramide and AUC levels (data not shown). Multiple regression analysis (using DAG, ceramide, and TG as independent variables) indicated that AUC levels were primarily predicted by DAG levels $(\beta=0.736$, $R^{2}$ change $\left.=0.541, P<0.001\right)$.

To explain the close relationship between intracellular DAG levels and insulin sensitivity, we investigated DAG-responsive PKC activity. We reasoned that decreases in steady-state levels of DAG would prevent overactivation of the conventional and novel isoforms of PKC. Overactivation of DAG-responsive PKC isoforms
Table 2

Fasting plasma levels of several adipokines and cytokines in 15-week-old male WT and MCKDgat1 mice after 8 weeks of HFD treatment

\begin{tabular}{lccccc}
\hline & $\begin{array}{c}\text { Adiponectin } \\
(\mu \mathbf{g} / \mathbf{m l})\end{array}$ & $\begin{array}{c}\text { IL-6 } \\
(\mathbf{n g} / \mathbf{m l})\end{array}$ & $\begin{array}{c}\text { TNF- } \alpha \\
(\mathbf{p g} / \mathbf{m l})\end{array}$ & $\begin{array}{c}\text { Resistin } \\
(\mathbf{n g} / \mathbf{m l})\end{array}$ & $\begin{array}{c}\text { RBP4 } \\
(\mu \mathbf{M})\end{array}$ \\
WT $(n=5-6)$ & $10.44 \pm 2.5$ & $0.88 \pm 0.86$ & $7.84 \pm 2.4$ & $2.05 \pm 0.50$ & $1.77 \pm 0.55$ \\
MCK-Dgat1 $(n=5-6)$ & $9.29 \pm 0.66$ & $0.22 \pm 0.23$ & $8.43 \pm 3.8$ & $2.10 \pm 0.46$ & $1.41 \pm 0.39$ \\
& $P=0.73$ & $P=0.14$ & $P=0.78$ & $P=0.86$ & $P=0.22$ \\
\hline
\end{tabular}

Data are expressed as mean \pm SD. 

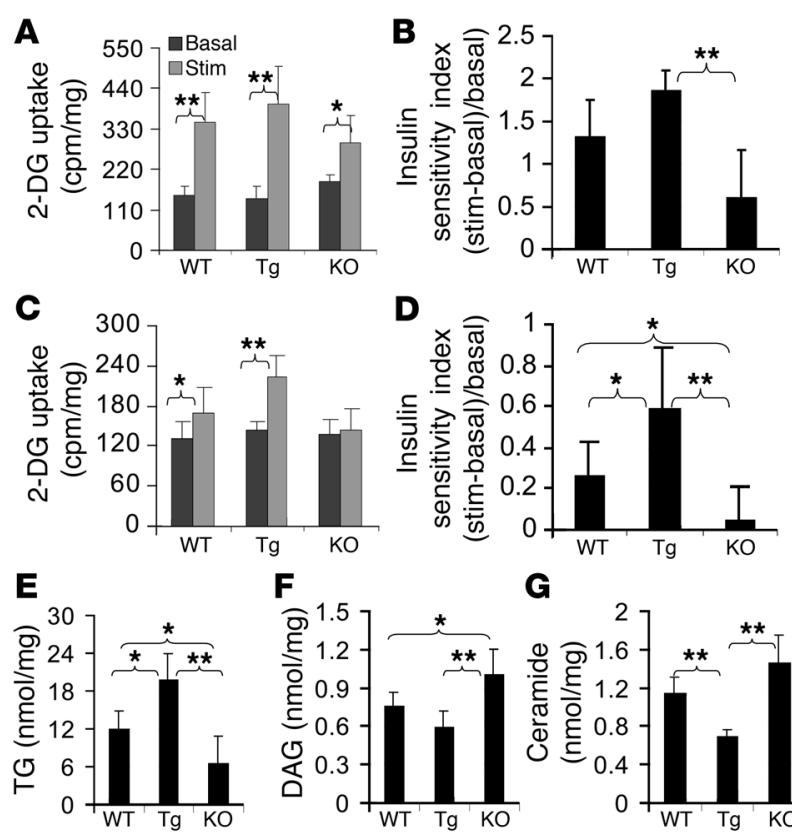

G

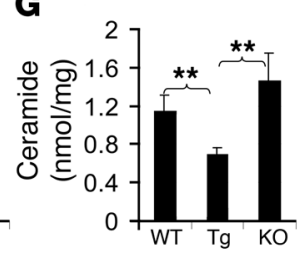

in muscle, including PKC $\beta,-\alpha,-\delta,-\varepsilon$, and $-\theta$, is linked to muscle insulin resistance $(19,53-58)$. In contrast to the conventional and novel PKC isoforms, atypical PKC isoforms do not respond to DAG, but rather they mediate insulin signaling (59). Since membrane localization is a measure of lipid-mediated activation of PKC $(53,54)$, we quantified membrane and cytosol fractions of PKC and calculated the percentage of membrane-bound PKC in each muscle specimen. The overall PKC activity (all isoforms combined) in the membrane fraction was increased in HFD-fed WT mice but remained low in transgenic Dgat 1 mice on the same HFD, as compared with WT mice on NC diet (Figure 5C). To distinguish the changes of DAG-responsive PKC from that of the atypical PKC, we then measured membrane-bound activities of representative PKC isoforms following immunoprecipitation with isoform-specific antibodies. Membrane PKC $\beta 2$, a major conventional PKC isoform in skeletal muscle, was increased in HFD-fed WT mice but not in HFD-fed Dgat 1 mice compared with NC-fed WT mice (Figure 5D). Similarly, lower activity levels of PKCE $(P=0.04)$ and PKC $\theta$ $(P=0.08)$ were also observed in the muscles of HFD-fed Dgat 1 mice compared with those of HFD-fed WT mice (Supplemental Figure 3). By contrast, the membrane fraction of atypical PKC $\lambda$ was not increased in HFD-fed WT mice but was increased in HFDfed Dgat 1 mice compared with NC-fed WT mice (Figure 5E). The percentage of membrane-bound activity for all PKC isoforms and for PKC $\beta 2$ showed the same patterns: low in NC-fed WT mice, higher in HFD-fed WT mice, and reverted to low in HFD-fed Dgat 1 mice (Figure 5F). A reversed pattern was seen for PKC $\lambda$, in that membrane-bound activity was higher in HFD-fed Dgat 1 mice than in HFD-fed WT mice (Figure 5F).

Potential downstream effectors of DAG-responsive PKC, JNK1, and insulin receptor substrate 1 (IRS-1) (19, 60-62) were then examined. JNK1 phosphorylation (Thr183/Tyr185) was increased in HFD-fed WT mice compared with WT mice on NC diet, whereas it remained low in HFD-fed Dgat1 mice (Figure 5G). JNK1 directly phosphorylates IRS-1 at Ser307 (62) and inhibits insulin responsiveness of PI3K and the downstream cascade (63). In agreement

\section{Figure 4}

Muscle insulin sensitivity and its response to FA challenges are DGAT1 genotype dependent. (A) Ex vivo basal and insulin-stimulated (Stim) 2-deoxyglucose (2-DG) uptake in isolated soleus muscles from WT $(n=4)$, MCK-Dgat1 transgenic $(n=6)$, and Dgat1-knockout $(n=4)$ mice without FA pretreatment. (B) Relative insulin-stimulated 2-DG uptakes as measured by the difference between insulin-stimulated and basal 2-DG uptakes divided by the basal uptake. Data are derived from the experiment described in A. (C) Ex vivo basal and insulin-stimulated 2-DG uptake in isolated soleus muscles from WT $(n=6)$, Dgat1 transgenic $(n=5)$, and Dgat1-knockout $(n=5)$ mice after the muscles were pretreated with $0.75 \mathrm{mM}$ FA for 2 hours. (D) Relative insulin-stimulated 2-DG uptakes were calculated as in $\mathbf{B}$; data are derived from the experiment described in C. TG $(\mathbf{E})$, DAG $(\mathbf{F})$, and ceramide $(\mathbf{G})$ contents in soleus muscles isolated from WT $(n=4)$, Dgat1 transgenic $(n=5)$, and Dgat1-knockout $(n=4)$ mice after the muscles were pretreated with $0.75 \mathrm{mM}$ FA for 2 hours. ${ }^{\star} P<0.05$; ${ }^{* *} P<0.01$.

with this, HFD feeding increased muscle phosphorylated IRS-1 (Ser307) levels in HFD-fed WT mice. However, this elevation was prevented in HFD-fed MCK-Dgat1 mice (Figure 5G), suggesting that modulation of IRS-1 is a key step in relaying information from the DAG-PKC pathway to the insulin signaling pathway. The effect of phosphorylation of IRS-1 at Ser307 on downstream signaling was further supported by the concordant change in the phosphorylation levels of the downstream effector Akt. Phosphorylated Akt (Ser473) in skeletal muscle of HFD-fed WT mice was significantly decreased compared with NC-fed WT mice, but this was reverted in HFD-fed Dgat 1 mice (Figure 5G). Unlike JNK1, IKK $\beta$, another target of PKC (64) that phosphorylates IRS-1 at Ser307 (65), appeared to be uninvolved in mediating the effect of DGAT1 in the transgenic muscle. The activity of IKK $\beta$ was assessed by first quantifying its downstream target, IкB- $\alpha$ and then by measuring nuclear NF- $\kappa \mathrm{B}$ activation. Upon phosphorylation by IKK $\beta$, IкB- $\alpha$

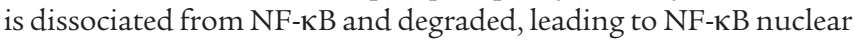
translocation and activation. No significant changes in IкB- $\alpha$ protein levels were evident among the 3 groups (Figure $5 \mathrm{H}$ ). Similarly, nuclear NF-кB levels determined by gel-shift assay also showed no difference between HFD-fed WT and Dgat 1 mice (Figure $5 \mathrm{H}$ ).

Finally, insulin responsiveness of Akt phosphorylation and GLUT4 membrane translocation were examined in the skeletal muscles of HFD-fed WT and Dgat1 mice. Upon insulin stimulation, levels of phosphorylated Akt and membrane-bound GLUT4 were both increased in the muscles of MCK-Dgat 1 mice and WT mice (Figure 6). However, the percent increases of insulin-stimulated Akt phosphorylation and GLUT4 membrane translocation were greater in HFD-fed Dgat1 mice compared with HFD-fed WT mice (266\% versus $85 \%$ for phosphorylated Akt and $225 \%$ versus $144 \%$ for membrane-bound GLUT4; Figure 6B). Thus, not only were basal levels of phosphorylated Akt and membrane-bound GLUT4 greater in MCK-Dgat1 muscles than in WT muscles, but also MCK-Dgat1 muscles were more responsive to insulin stimulation.

\section{Discussion}

The present study shows that exercise increased DGAT1 expression and total DGAT activity and that transgenic expression of DGAT1 in mouse skeletal muscle recapitulated the exercise effect (the athlete's paradox) without exercise training. Myocellular overexpression of DGAT1 resulted in increased IMTG levels but decreased myocellular DAG and ceramide levels and improved muscle and whole-body insulin sensitivity. The greater insulin 


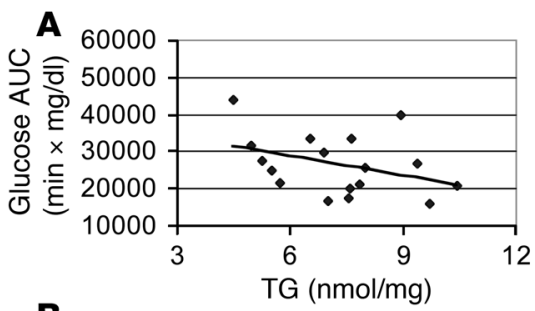

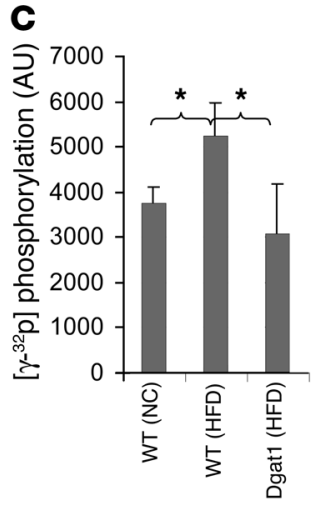

All PKCs-Mb

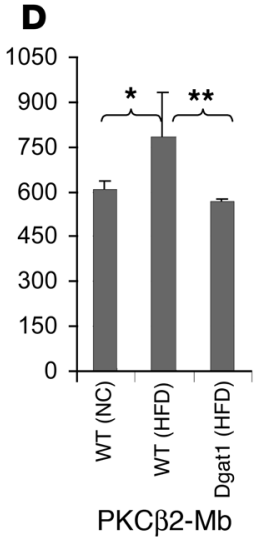

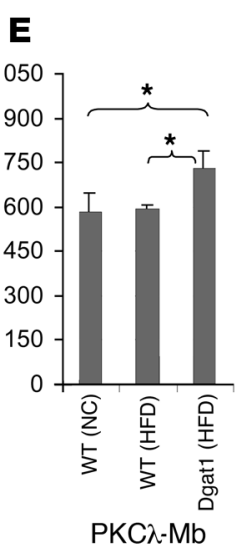

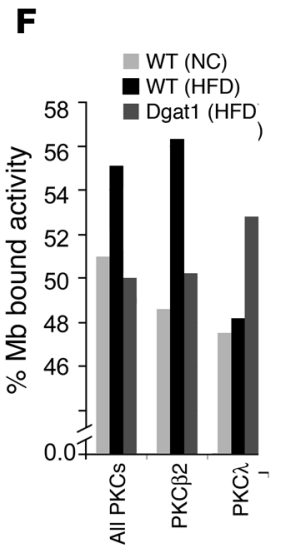

\section{G}
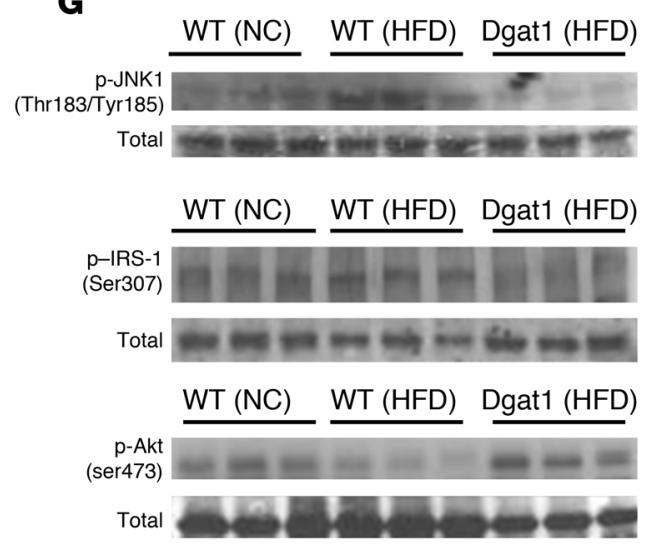

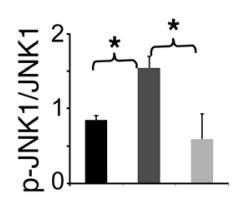

H
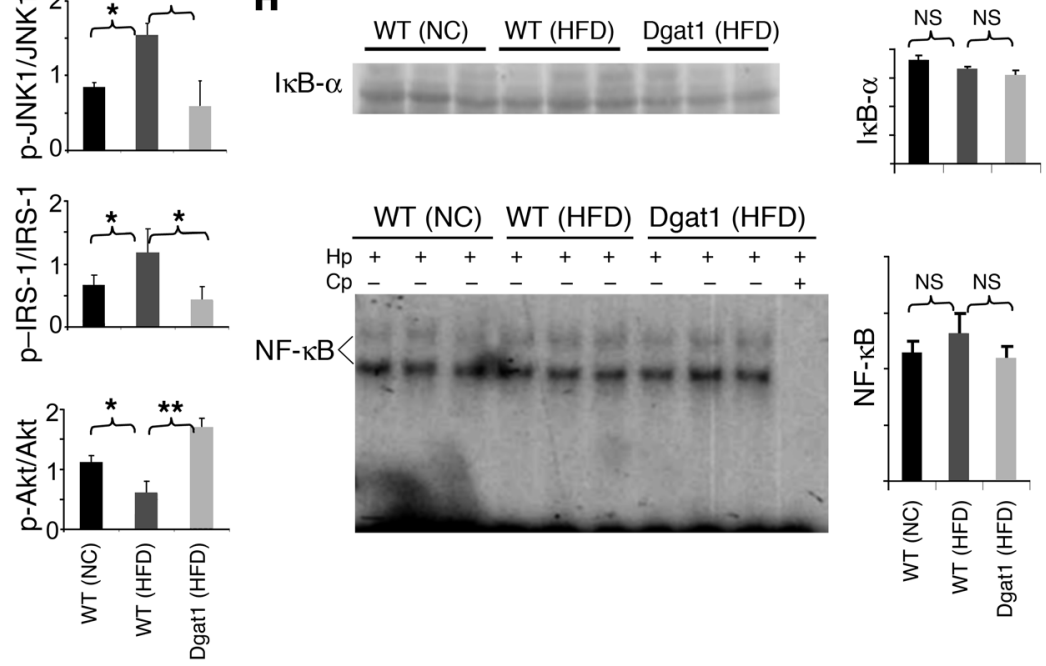

Figure 5

DGAT1-mediated protection against FA-induced insulin resistance in skeletal muscle involves DAG-PKC and its downstream signal transduction. (A) An inverse correlation was found between muscle TG levels with glucose AUC levels in GTT. Adjusted $R^{2}=0.126 ; P=0.084$. (B) Positive correlation between muscle DAG levels with glucose AUC levels. Adjusted $R^{2}=0.515 ; P=0.001$. Data in A and B are from NC-fed and HFDfed MCK-Dgat1 mice. (C-E) Membrane-bound PCK activity levels of all isoforms combined (C), the conventional isoform PKC $\beta 2$ (D), and the atypical isoform PKC $\lambda(E)$ in soleus muscles from NC-fed WT mice $(n=4)$, HFD-fed WT mice $(n=4)$, and HFD-fed transgenic mice $(n=4)$. (F) Percentages of membrane-bound PCK activity levels of all isoforms combined, PKC $\beta 2$, and PKC $\lambda$ in NC-fed WT, HFD-fed WT, and HFD-fed Dgat1 mice. (G) Western blots for total and phosphorylated JNK1 ( $p$-JNK1; Thr183/Tyr185), total and phosphorylated IRS-1 ( $p-I R S-1$; Ser307), and total and phosphorylated Akt (p-Akt; Ser473) in muscle specimens from NC-fed WT, HFD-fed WT, and HFD-fed Dgat1 mice, as indicated. Densitometry was used to quantify phosphorylated proteins (in arbitrary units) as shown at right ( $n=3$ in each group). (H) Assessment of the IKK $\beta-N F-\kappa B$ pathway. Top: Western blot for muscle IкB- $\alpha$ levels from WT mice on NC diet, WT mice on HFD, and Dgat1 mice on HFD. Bottom: EMSAs for muscle NF-кB activity levels in NC-fed WT, HFD-fed WT, and HFD-fed Dgat1 mice. hp, hot probe; cp, cold probe; NS, no statistical significance. Densitometric quantification (in arbitrary units) is shown at right ( $n=3$ in each group). ${ }^{*} P<0.05 ;{ }^{* *} P<0.01$.

sensitivity in exercise-trained muscle has been attributed in part to increased FA $\beta$-oxidation capacity $(22,31,32)$. Our data indicate that augmenting TG synthesis in skeletal muscle is sufficient to increase insulin sensitivity. Thus, insulin sensitivity in skeletal muscle is intrinsically connected with myocellular DGAT1 via its effect on intramyocellular FA metabolites.

The effect of DGAT1 on levels of myocellular lipids and insulin sensitivity was further verified in isolated muscles from DGAT1knockout mice, DGAT1-transgenic mice, and WT mice. Our results indicate that the levels of DGAT1 determine muscle insulin sensitivity and susceptibility to FA-induced insulin resistance - while DGAT1 overexpression enhanced muscle insulin sensitivity and lessened FA-induced insulin resistance in soleus muscles, DGAT1 deficiency was associated with diminished insulin sensitivity and rendered the muscles completely vulnerable to FA challenges. The finding in skeletal muscles isolated from Dgat $1^{-/-}$mice appears to differ from the phenotype of the whole-body animal but is not unexpected. Dgat $1^{-/-}$mice have decreased adiposity and are resistant to HFD-induced obesity as a result of increased whole-body energy expenditure associated with increased locomotor activities $(48,66,67)$. Although Dgat $1^{-/-}$mice also exhibited a greater muscle insulin sensitivity, this was likely caused by transacting insulin-sensitizing adipokine(s) secreted from the DGAT1-deficient adipose tissue (68). The importance of the altered adipokine 


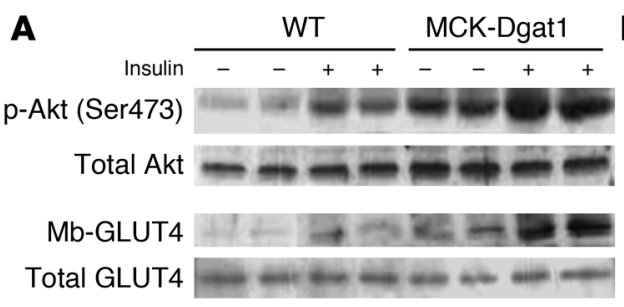

\section{Figure 6}

Insulin-induced Akt phosphorylation and GLUT4 membrane translocation in muscles from MCK-Dgat1 and WT mice. (A) Levels of total Akt, phosphorylated Akt (Ser473), total GLUT4, and membrane-bound GLUT4 in muscle specimens from HFD-fed WT and MCK-Dgat1 transgenic mice. Muscles were incubated in the presence (+) or absence $(-)$ of $100 \mathrm{nM}$ insulin for 30 minutes before tissue homogenates or membrane fractions were prepared and analyzed by Western blot. (B) Ratios of phosphorylated Akt to total Akt and membrane-bound GLUT4 to total GLUT4 in WT and MCK-Dgat1 muscles at the basal and insulin-stimulated states. The values are means from 2 experiments. Percent increases shown in each panel for insulin-stimulated Akt phosphorylation or GLUT4 membrane translocation were calculated as (insulin - basal)/basal.

functions in these mice was demonstrated by transplantation of fat from Dgat $1^{-/}$mice to WT mice, which resulted in increased insulin sensitivity in the recipient mice (69). The result of our study is reminiscent of the study by Listenberger et al. in cultured cells, in which promotion of FA-to-TG conversion lessened FAinduced lipotoxicity instead of increasing it (70). The dissociation of increased IMTG from insulin resistance is also supported by findings in the recently created adipose TG lipase-knockout (ATGL-knockout) mice. ATGL is required for the initial conversion of TG to DAG during lipolysis. ATGL $/$ - mice exhibit a greater glucose tolerance in skeletal muscle despite a marked accumulation of IMTG (71).

Taken together, the evidence supports our working model depicted in Figure 6. In obesity, there is increased FA influx to skeletal muscle, which causes increased IMTG levels as well as levels of other intracellular lipotoxic FA derivatives (such as DAG and ceramide), leading to insulin resistance (Figure 7A). In exercised muscle, however, because of upregulation of DGAT1, the TG synthesis capacity is increased, resulting in more FA substrates channeled to storage in the form of TG and in less to synthesis of lipotoxic FA-derivatives (Figure 7B). Thus, while an increase in IMTG levels in obesity serves as a marker for FA overload, an increase in IMTG levels due to exercise indicates an increased capacity for TG synthesis and storage. This exercise-induced change is analogous to an increase in the "buffering capacity" of adipose tissue, through which fluxes of intracellular FA can be better handled, particularly at times when FA overload occurs.

In MCK-Dgat 1 mice, the reduction of HFD-induced insulin resistance appears to be accounted for at least in part by attenuation of fat-induced activation of DAG-responsive PKCs. The levels of membrane-bound PKC activity (of all the DAG-responsive isoforms combined) in the HFD-fed transgenic mice were lower than in HFD-fed WT mice but were comparable to the levels in NC-fed WT mice; measurements of specific DAG-responsive PKC isoforms $\beta 2$, $\varepsilon$, and $\theta$ confirmed these results. DAG is an allosteric activator of the conventional and novel isoforms of PKC. Persistent activation of DAG-responsive PKC isoforms such as PKC $\beta,-\alpha,-\delta$, $-\varepsilon$, and $-\theta$ in muscle are associated with muscle insulin resistance $(19,53-57)$. Deletion of PKC $\theta$ in mice results in protection against muscle insulin resistance induced by lipid infusion (58), although both transgenic mice with muscle-specific expression of dominant- negative PKC $\theta$ and PKC $\theta$-knockout mice exhibit age-associated or diet-associated obesity and whole-body insulin resistance $(72,73)$.

Although elevated DAG may not always enable activation of PKCs (if permissive levels of phosphatidylserine and fatty acyl-CoA are absent; ref. 74) or impair insulin signaling, decreases in DAG levels would be expected to attenuate many overactivated, DAGresponsive isoforms of $\mathrm{PKC}$ and protect muscle from FA-induced insulin resistance. The close association between decreases in DAG levels and the activities of DAG-responsive PKC in MCK-Dgat1 mice supports this concept. Furthermore, we showed that reduced

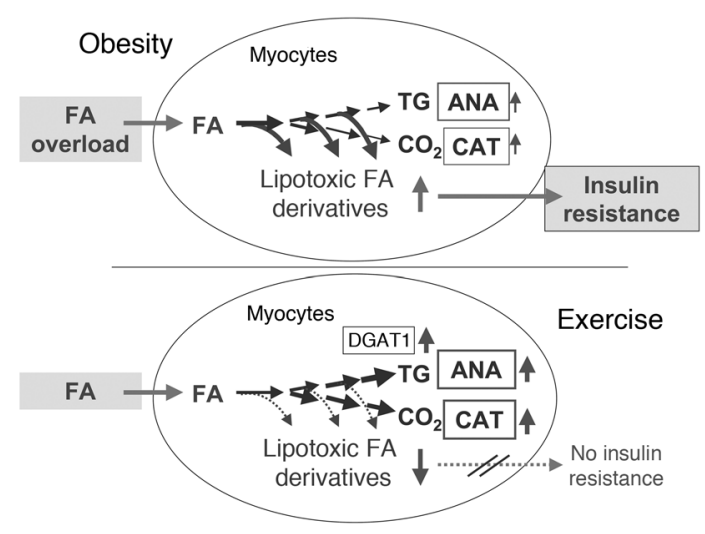

\section{Figure 7}

Models for fat-induced insulin resistance in obesity and protection by exercise-induced augmentation of TG synthesis. This model assumes that excessive accumulation of certain intramyocellular FA-derived lipid metabolites is lipotoxic and is a cause for muscle insulin resistance. In this model, both the terminal catabolic pathway (CAT) of FA oxidation and the anabolic pathway (ANA) of TG synthesis are viewed as beneficial in removing potentially lipotoxic FA derivatives from myocytes. In obesity, the primary driving force for increased myocellular TG levels is FA overload of myocytes. Because of the increased intracellular FA substrates, not only are TG levels increased, but levels of lipotoxic FA derivatives are also increased, leading to muscle insulin resistance. Exercise upregulates DGAT1, which increases the TG synthesis capacity. This also increases TG formation but reduces FA substrate levels, resulting in decreased formation of lipotoxic FA derivatives. Thus, upregulation of myocellular DGAT1 is protective against FA-induced insulin resistance. 
DAG and PKC levels were associated with reduced activity of JNK, a downstream target of the DAG-responsive PKC isoforms $\beta$ and $\theta$ in leukocytes $(60,61)$. Phosphorylation of IRS-1 at Ser307 catalyzed directly by JNK has been demonstrated (62). The reduced serine phosphorylation of IRS-1 makes this protein a better substrate for insulin-stimulated tyrosine phosphorylation (62), which in turn leads to a greater activation of downstream effectors in the insulin signaling cascade, including increased phosphorylation of Akt and membrane translocation of GLUT4 as demonstrated in this study.

It is worth noting that the increases in DGAT1 mRNA and DGAT activity levels are greater in the muscle of transgenic mice (2- to 3-fold increases) than in the muscle of exercise-trained mice ( $\sim 70 \%$ increases). Interestingly, the magnitudes of changes in muscle lipid levels do not exactly parallel those in DGAT activity in magnitudes; the levels of decreases in muscle DAG and ceramide contents are, in fact, very similar between the transgenic model and the exercised mice. The discordance between changes in DGAT1 and DAG levels was also observed in cultured C2C12 myotubes transduced by DGAT1 recombinant adenovirus. In that case, DGAT activity levels were increased 4- to 5-fold, while decreases in DAG levels were still no greater than 50\%. Thus, it appears that muscle DAG and ceramide levels are subjected to more rigorous homeostatic regulations than are DGAT1 levels. Very similar levels of decreases in muscle DAG and ceramide contents have also been described in exercised humans. In a recent report by Bruce et al., a $15 \%$ reduction in DAG and a $42 \%$ reduction in ceramide levels are found in skeletal muscles of obese humans after endurance training (32). The similar magnitudes of decreases in muscle DAG and ceramide contents in MCK-Dgat1 mice and in exercised animals and humans suggest that the MCK-Dgat1 model is physiologically relevant in this regard.

Yu et al. reported that muscle insulin resistance induced by lipid infusion in rats is only associated with increased myocellular fatty acyl-CoA and DAG levels but not with increased TG or ceramide levels (19). Rats fed a HFD (53\% fat calories) also exhibit insulin resistance that is associated with increased DAG and TG levels but an unchanged ceramide content in skeletal muscle (20). While these results raise the question of whether ceramide plays a major role in mediating fat-induced muscle insulin resistance in vivo, our data show that HFD feeding increases both DAG and ceramide levels in skeletal muscle and that lower levels of these lipids are associated with greater insulin sensitivity in MCK-Dgat1 mice. In addition to the DAG-mediated effect on insulin signaling as already discussed, a reduction of myocellular ceramide levels would be expected to increase phosphorylation of Akt, and therefore insulin sensitivity, as ceramide inhibits Akt phosphorylation $(75,76)$. Nevertheless, whether decreased ceramide levels in MCKDgat 1 mice contribute to the improved muscle insulin sensitivity cannot be determined in the present study, as validation of the true effect of decreased ceramide levels would require that muscle DAG levels be independently controlled or normalized.

Although the MCK promoter would be expected to direct greater expression of the transgene in glycolytic myofibers than in oxidative myofibers or cardiac myofibers, the relative differences among these fibers may be small and may not be exactly as predicted (77). Our transgenic mice do have much weaker expression of the transgene in the heart than in the skeletal muscle. However, the differences between the fiber types within skeletal muscle appear insignificant, as similarly high expression levels of the transgene were detected in various muscle groups with different fiber composi- tions. This suggests that the effects of DGAT1 in these transgenic mice are derived from both type I and type II myofibers.

In conclusion, this study identifies DGAT1 as an important mediator in exercise-induced increase of IMTG levels and improvement of insulin sensitivity in skeletal muscle. Our data indicate that upregulation of myocellular DGAT1 augments TG synthesis and protects skeletal muscle against fat-induced insulin resistance. Thus, increasing muscle DGAT activity is theoretically a possible means to prevent and treat insulin resistance and type 2 diabetes mellitus.

\section{Methods}

DGAT1 recombinant adenovirus and cell culture. The DGAT1 recombinant adenovirus was prepared via homologous recombination between pAd. CMV-link.1-hDGAT1 and the ClaI-digested defective adenoviral DNA in 293 cells as previously described (78). C2C12 myoblasts were cultured in high glucose (25 mM) DMEM with 10\% FBS. Medium was changed every other day, and cells were split and passed before they reached confluence. $\mathrm{C} 2 \mathrm{C} 12$ cells were differentiated in the DMEM supplemented with $2 \%$ horse serum after reaching confluence. For viral transduction, the DGAT1 recombinant adenovirus was delivered to $\mathrm{C} 2 \mathrm{C} 12$ myotubes on day 5 following differentiation, at a dose of $5 \mathrm{pfu} / \mathrm{cell}$ (78). For controls, cells were either mock transduced or infected with a GFP-recombinant adenovirus. Assays were carried out 72 hours after viral infection.

Mice, diets, and exercise. Animal protocols were in compliance with accepted standards of animal care and were approved by the Columbia University Institutional Animal Care and Use Committee. Transgenic mice were produced by pronuclear injection (79) of C57BL/6 blastocysts with the MCKDgat 1 transgene (Figure 2A). Male mice were used in experiments unless otherwise indicated. Wild-type C57BL/6J mice and DGAT1-knockout C57BL/6J mice (48) were purchased from The Jackson Laboratory. Mice were housed in a barrier facility with 12-hour light/12-hour dark cycles and had ad libitum access to either NC (5053 PicoLab Rodent Diet 20; Purina Mills) or HFD (45\% fat primarily from lard, $20 \%$ protein, $35 \%$ carbohydrates, catalog no. D12451; Research Diets Inc.). Timing and duration of HFD treatments are as indicated in Results and the legend for Figure 3. WT male mice were exercised by placement in a basin containing water at $35^{\circ} \mathrm{C}$. Swimming was supervised with intermittent hand propulsion to prevent resting. Mice were subjected to 6 swimming sessions each day for 1 week, with each session lasting 5-10 minutes (mice were taken out if heads bobbed). Both the control group (sedentary) and the swimming group were fed HFD during the week of study.

Fasting plasma parameters. Plasma specimens were prepared from bloods drawn from mice after overnight fasting (10 pm to 8:30 am), frozen in liquid nitrogen, and stored at $-80^{\circ} \mathrm{C}$ until they were assayed for glucose and adipokine levels. Plasma adiponectin levels were measured using Mouse Adiponectin Single-Plex Kit (Millipore), and plasma levels of insulin, IL-6, TNF- $\alpha$, and resistin were measured using Mouse Serum Adipokine LINCOplex Kit (Millipore) by the Mouse Metabolic Phenotyping Center at Vanderbilt University (Nashville, Tennessee, USA). Plasma RBP4 levels were determined by radioimmunoassay as previously described (80).

Intraperitoneal GTT and ITT. GTT were carried out after a 6-hour fast (from 8 am to $4 \mathrm{pm}$ ). Glucose ( $1 \mathrm{~g} / \mathrm{kg}$ body weight) was administered by intraperitoneal injection. Blood samples were obtained from tail veins before injection ( $0 \mathrm{~min}$ ), and 30, 60, and 120 minutes after injection, and blood glucose levels were determined by glucometry (Ascensia ELITE Blood Glucose Meter; Bayer). ITT was carried out also after 6-hour fasting with intraperitoneal injection of human insulin $(0.5 \mathrm{U} / \mathrm{kg}$ body weight), and blood glucose levels were measured at $0,15,30,60$, and 90 minutes after injection. 
Ex vivo muscle 2-deoxyglucose uptake. Muscle 2-deoxyglucose uptake was carried out in isolated intact soleus muscles as described in ref. 79. Pairs of soleus muscles were isolated from overnight-fasted mice. One muscle was used for basal uptake and the other for insulin-stimulated uptake. Isolated soleus muscles were first washed for 1 hour at $35^{\circ} \mathrm{C}$ in Krebs-Henseleit bicarbonate buffer (KHB; $10 \mathrm{mM}$ HEPES, pH 7.35, $24 \mathrm{mM} \mathrm{NaHCO}_{3}, 114 \mathrm{mM} \mathrm{NaCl}$, $5 \mathrm{mM} \mathrm{KCl}, 1 \mathrm{mM} \mathrm{MgCl}_{2}$, and $2.2 \mathrm{mM} \mathrm{CaCl}_{2}$ ) containing $8 \mathrm{mM}$ glucose, 32 $\mathrm{mM}$ mannitol, and $0.1 \% \mathrm{BSA}$. The muscles were then incubated for 2 hours at $35^{\circ} \mathrm{C}$ in $\mathrm{KHB}$ containing $8 \mathrm{mM}$ glucose, $32 \mathrm{mM}$ mannitol, and $4 \%$ BSA either in the presence or absence of $0.375 \mathrm{mM}$ each palmitate and oleate. Incubation was carried out with constant shaking and continuous gassing with $95 \%$ $\mathrm{O}_{2} / 5 \% \mathrm{CO}_{2}$. Muscles were then washed for 15 minutes in $\mathrm{KHB}$ containing $40 \mathrm{mM}$ mannitol and $0.1 \% \mathrm{BSA}$, followed by incubation in the same buffer in the absence (basal) and presence (stimulation) of $100 \mathrm{nM}$ insulin at $35^{\circ} \mathrm{C}$ for 30 minutes. Uptake of 2-deoxy-D-[1-3 $\mathrm{H}]$ glucose was carried out in $1 \mathrm{mM}$ glucose, $39 \mathrm{mM}$ mannitol, and $\left[{ }^{14} \mathrm{C}\right]$ mannitol tracer used to control for nonspecific radioactivity trapping in the extracellular spaces of the muscle. After subtracting nonspecifically trapped radioactivity, the uptake of 2-deoxy-D$\left[1-{ }^{3} \mathrm{H}\right]$ glucose was expressed as $\left[{ }^{3} \mathrm{H}\right]$ counts per milligram of tissue.

RT-PCR. RNA isolation, reverse transcription using random hexamer primers, and subsequent RT-PCR (DNA Engine Opticon 2 Real-Time PCR Detection System; Bio-Rad) were all carried out as previously described (79). Total DGAT1 and DGAT2 mRNA levels were quantified using the following primers: DGAT1, forward, 5'-GTGCACAAGTGGTGCATCAG-3', reverse, 5'-CAGTGGGATCTGAGCCATCA-3'; DGAT2, forward, 5'-CAGCAAGAAGTTTCCTGGCAT-3', reverse, 5'-CCTCCCACCACGATGATGAT-3'; mRNA levels were then expressed as ratios relative to $\beta$-actin.

Muscle DGAT activity and TG, DAG, and ceramide contents. DGAT activity was measured in vitro in membrane fractions isolated from muscle specimens using $\left[{ }^{14} \mathrm{C}\right]$-labeled palmitoyl CoA as previously described (78). The amounts of both DAG and fatty acyl-CoA in the reaction mixture were in excess, and the reaction rate was of the first order, with respect to the amount of input DGAT1 activity to be assayed. $\mathrm{MgCl}_{2}$ at a concentration of $150 \mathrm{mM}$ was used in this study. To measure TG, DAG, and ceramide, lipids were first extracted from muscles using chloroform/methanol/HCl (79). Butylated hydroxytoluene $(0.01 \%)$ was included in the extraction solution as an antioxidant, and $\left[{ }^{3} \mathrm{H}\right]$ triolein $(0.25 \mu \mathrm{Ci})$ was used as an internal control for TG recovery. TG mass in lipid extracts was determined enzymatically with a colorimetric kit (Sigma-Aldrich). DAG and ceramide levels were measured using a DAG kinase-based method, in which DAG and ceramide are quantitatively phosphorylated to form ${ }^{32} \mathrm{P}$-labeled phosphatidic acid and ceramide-1-phosphate, respectively, that are then quantified. Lipids extracted from muscle specimens were dried under $\mathrm{N}_{2}$ stream, redissolved in $7.5 \%$ octyl- $\beta$ - D-glucoside containing $5 \mathrm{mM}$ cardiolipin and $1 \mathrm{mM}$ diethylenetriamine pentaacetate. Reaction was carried out at room temperature for 30 minutes in $100 \mathrm{mM}$ imidazole. $\mathrm{HCl}, 100 \mathrm{mM} \mathrm{NaCl}, 25 \mathrm{mM} \mathrm{MgCl} 2,2 \mathrm{mM}$ EGTA (pH 6.6), $2 \mathrm{mM} \mathrm{DTT}, 10 \mu \mathrm{g} / 100 \mu \mathrm{l}$ DAG kinase (Sigma-Aldrich), $1 \mathrm{mM} \mathrm{ATP}$, and $1 \mu \mathrm{Ci} / 100 \mu \mathrm{l}\left[\gamma^{-32} \mathrm{P}\right] \mathrm{ATP}$. The reaction was stopped by addition of chloroform/methanol $(\mathrm{v} / \mathrm{v}, 2: 1)$ and $1 \% \mathrm{HClO}_{4}$, and lipids were extracted and washed twice with $1 \% \mathrm{HClO}_{4}$. Lipids were resolved by TLC (Partisil K6 adsorption TLC plates, catalog no. LK6D; Whatman; mobile phase containing chloroform/methanol/acetic acid, $\mathrm{v} / \mathrm{v}, 65: 15: 5$ ). The bands corresponding to phosphatidic acid and ceramide-1-phosphate were identified and silicon scraped into a scintillation vial for radioactivity measurement. $\left[{ }^{3} \mathrm{H}\right]$ triolein bands from the same TLC plates were also identified and quantified in the same way and were used as controls for lipid recovery.

$P K C$ activity. Assays were performed via a reaction that measures the transfer of ${ }^{32} \mathrm{P}$ from $\left[\gamma^{-32} \mathrm{P}\right]$ ATP to a PKC-specific substrate peptide by PKC in muscle homogenates using a PKC assay kit from Millipore. Membrane and cytosol PKCs (all isoforms) were measured in the presence of a PKA inhibitor peptide, R34571, and a cocktail of PKC activators including phosphatidylserine and DAG. To measure activity levels of specific isoforms of PKC, the isoforms were first immunoprecipitated using specific antibodies, followed by the PKC activity assays in the presence (PKC $\beta 2, \mathrm{PKC} \varepsilon$, and $\mathrm{PKC} \theta$ ) or absence (PKC $\lambda$ ) of lipid activators. Quantification of the phosphorylated substrate peptides was carried out by scintillation counting on P81 phosphocellulose paper after 3 washes with $0.75 \%$ phosphoric acid followed by an acetone wash.

Western blot. Cell lysates, membrane, and cytosol fractions were prepared using Immunoprecipitation Kit (Roche Applied Science). A total of 20 to $30 \mathrm{mg}$ tissues were homogenized in lysis buffer. Total cell lysates were obtained from the postmitochondrial supernatants after centrifugation at $15,000 \mathrm{~g}$ for 10 minutes at $4^{\circ} \mathrm{C}$. To obtain membrane and cytosol fractions, muscle tissues were homogenized in the homogenization buffer containing 20 mM HEPES, pH 7.4, $1 \mathrm{mM} \mathrm{CaCl}_{2}, 1 \mathrm{mM} \mathrm{MgCl}_{2}, 5 \mathrm{mM} \mathrm{Na}_{3} \mathrm{VO}_{4}, 10 \mathrm{mM}$ $\mathrm{NaF}, 1 \mathrm{mM}$ DTT, and a cocktail of protease inhibitors. One-fourth volume of $30 \%$ sucrose was added to the samples immediately following homogenization. The mixtures were then centrifuged at $1,500 \mathrm{~g}$ for 10 minutes at $4^{\circ} \mathrm{C}$. The supernatants were collected and centrifuged at $150,000 \mathrm{~g}$ for 1 hour at $4^{\circ} \mathrm{C}$. The membrane pellets were homogenized and resuspended in a 400- $\mu$ l buffer containing $20 \mathrm{mM}$ HEPES, pH 7.4, $0.25 \mathrm{M}$ sucrose, $5 \mathrm{mM}$ $\mathrm{Na}_{3} \mathrm{VO}_{4}, 10 \mathrm{mM} \mathrm{NaF}, 1 \mathrm{mM}$ DTT, and protease inhibitors. The cytosol fraction was obtained by concentrating the supernatants by acetone precipitation. Proteins ( $50 \mu \mathrm{g}$ per sample) were resolved by SDS-PAGE and then transferred onto nitrocellulose membrane. Immunoblotting was carried out using the following primary antibodies: anti-PKC $\beta 2$, anti-JNK1 (Santa Cruz Biotechnology Inc.), anti-p-Akt (Ser473), anti-p-IRS-1 (Ser307), anti-p-JNK/stress-activated protein kinase (Thr183/Tyr185) (Cell Signaling Technology), anti-GLUT1, and anti-GLUT4 (Millipore), as indicated in Results and Figures 3, 5, and 6. Immunoblots were developed using an ECL Advance Western Blotting Detection Kit (Amersham Biosciences).

EMSA. The assay for NF-KB was performed as previously described (81). Cell extracts were made with a Nuclear Extract Kit from Active Motif. Double-stranded NF- $\kappa B$ oligonucleotides (5'-AGTTGAGGGGACTTTCCCAGGC- $\left.3^{\prime}\right)$ were end labeled with $\left[\gamma^{-32} \mathrm{P}\right]$ ATP using T4 Polynucleotide Kinase (Promega). Cell extracts (20 $\mu$ g each sample) were preincubated at room temperature for 15 minutes in $10 \mathrm{mM}$ Tris- $\mathrm{HCl}, \mathrm{pH} 7.5,1 \mathrm{mM} \mathrm{MgCl}_{2}$, $0.5 \mathrm{mM}$ EDTA, $0.5 \mathrm{mM}$ DTT, $50 \mathrm{mM} \mathrm{NaCl}, 0.05 \mathrm{mg} / \mathrm{ml}$ poly $(\mathrm{dI}-\mathrm{dC})$, and $4 \%(\mathrm{v} / \mathrm{v})$ glycerol, followed by another 20 minutes of incubation with a $\left[{ }^{32} \mathrm{P}\right]$-labeled NF- $\mathrm{BB}$ oligonucleotide probe. In competition experiments, a cold NF- $\kappa B$ probe $(1 \mathrm{pmol} / 20 \mu \mathrm{l})$ was added to the reaction mixture. The reaction mixture was subjected to electrophoresis in polyacrylamide gels in $50 \%$ concentration of Tris-borate-EDTA buffer. The bound and free probes were visualized by autoradiography.

Histology. Muscles were isolated from mice fasted for 12 hours and were embedded in a Tissue-Tek OCT compound (Sakura Finetek). Tissue sections (7- $\mu \mathrm{m}$ sections) were stained with oil red $\mathrm{O}$ and counterstained with $\mathrm{H} \& \mathrm{E}$.

Statistics. Data were expressed as mean \pm SD. Statistical differences were first analyzed by ANOVA to determine overall treatment effects (genotype effects included), followed by post-hoc comparisons between concerned study groups (Statistica version 6.0; StatSoft). A 2-tailed $P$ value of less than 0.05 was used to determine statistical significance. Multiple regression analyses were performed to show correlations between muscle lipids and insulin sensitivity. The forward and backward stepwise regression analyses were used to assess independent effects and contributions of different muscle lipids to muscle insulin sensitivity.

\section{Acknowledgments}

We thank Ira Goldberg and Henry Ginsberg for discussions and reviewing the manuscript. We would also like to thank William 
Blaner and Nuttaporn Wongsiriroj for giving us DGAT1-knockout mice and for measuring plasma RBP4 levels. We also acknowledge the Columbia University Diabetes and Endocrinology Research Center facility for providing technical assistance and the Mouse Metabolic Phenotyping Center at Vanderbilt University for measuring plasma adipokine levels. This study was supported by grants DK60530, DK071866, DK063034, and HL073029 from the NIH.
Received for publication October 6, 2006, and accepted in revised form March 12, 2007.

Address correspondence to: Yi-Hao Yu, Division of Preventive Medicine, Department of Medicine, Columbia University, 630 W. 168th Street, PH 10-305J, New York, New York 10032, USA. Phone: (212) 305-2068; Fax: (212) 305-3213; E-mail: yy102@columbia.edu.
1. Unger, R.H. 2002. Lipotoxic diseases. Annu. Rev. Med. 53:319-336.

2. Yu, Y.H., and Ginsberg, H.N. 2004. The role of acyl-CoA:diacylglycerol acyltransferase (DGAT) in energy metabolism. Ann. Med. 36:252-261.

3. Phillips, D.I., et al. 1996. Intramuscular triglyceride and muscle insulin sensitivity: evidence for a relationship in nondiabetic subjects. Metabolism. 45:947-950.

4. Ebeling, P., et al. 1998. Intramuscular triglyceride content is increased in IDDM. Diabetologia. 41:111-115.

5. Manco, M., et al. 2000. Insulin resistance directly correlates with increased saturated fatty acids in skeletal muscle triglycerides. Metabolism. 49:220-224.

6. Borkman, M., et al. 1993. The relation between insulin sensitivity and the fatty-acid composition of skeletal-muscle phospholipids. N. Engl. J. Med. 328:238-244.

7. Pan, D.A., et al. 1997. Skeletal muscle triglyceride levels are inversely related to insulin action. Diabetes. 46:983-988.

8. Perseghin, G., et al. 1999. Intramyocellular triglyceride content is a determinant of in vivo insulin resistance in humans: a $1 \mathrm{H}-13 \mathrm{C}$ nuclear magnetic resonance spectroscopy assessment in offspring of type 2 diabetic parents. Diabetes. 48:1600-1606.

9. Virkamaki, A., et al. 2001. Intramyocellular lipid is associated with resistance to in vivo insulin actions on glucose uptake, antilipolysis, and early insulin signaling pathways in human skeletal muscle. Diabetes. 50:2337-2343.

10. Krssak, M., et al. 1999. Intramyocellular lipid concentrations are correlated with insulin sensitivity in humans: a $1 \mathrm{H}$ NMR spectroscopy study. Diabetologia. 42:113-116.

11. Rosa, G., et al. 2003. Decreased muscle acetyl-coenzyme A carboxylase $2 \mathrm{mRNA}$ and insulin resistance in formerly obese subjects. Obes. Res. 11:1306-1312.

12. Heron-Milhavet, L., et al. 2004. Muscle-specific overexpression of CD36 reverses the insulin resistance and diabetes of MKR mice. Endocrinology. 145:4667-4676.

13. Shimomura, I., et al. 1999. Leptin reverses insulin resistance and diabetes mellitus in mice with congenital lipodystrophy. Nature. 401:73-76.

14. Oral, E.A., et al. 2002. Leptin-replacement therapy for lipodystrophy. N. Engl. J. Med. 346:570-578.

15. Qu, X., Seale, J.P., and Donnelly, R. 1999. Tissue and isoform-selective activation of protein kinase $\mathrm{C}$ in insulin-resistant obese Zucker rats - effects of feeding. J. Endocrinol. 162:207-214.

16. Ellis, B.A., et al. 2000. Long-chain acyl-CoA esters as indicators of lipid metabolism and insulin sensitivity in rat and human muscle. Am. J. Physiol. Endocrinol. Metab. 279:E554-E560.

17. Kim, J.K., et al. 2001. Tissue-specific overexpression of lipoprotein lipase causes tissue-specific insulin resistance. Proc. Natl. Acad. Sci. U. S. A. 98:7522-7527.

18. Franch, J., et al. 2002. Acyl-CoA binding protein expression is fiber type-specific and elevated in muscles from the obese insulin-resistant Zucker rat. Diabetes. 51:449-454.

19. Yu, C., et al. 2002. Mechanism by which fatty acids inhibit insulin activation of insulin receptor substrate-1 (IRS-1)-associated phosphatidylinositol 3-kinase activity in muscle. J. Biol. Chem. 277:50230-50236.
20. Lee, J.S., et al. 2006. Saturated, but not n-6 polyunsaturated, fatty acids induce insulin resistance: role of intramuscular accumulation of lipid metabolites. J. Appl. Physiol. 100:1467-1474.

21. Hoppeler, H., et al. 1985. Endurance training in humans: aerobic capacity and structure of skeletal muscle. J. Appl. Physiol. 59:320-327.

22. Goodpaster, B.H., et al. 2001. Skeletal muscle lipid content and insulin resistance: evidence for a paradox in endurance-trained athletes. J. Clin. Endocrinol. Metab. 86:5755-5761.

23. Schenk, S., et al. 2005. Postexercise insulin sensitivity is not impaired after an overnight lipid infusion. Am. J. Physiol. Endocrinol. Metab. 288:E519-E525.

24. Decombaz, J., et al. 2001. Postexercise fat intake repletes intramyocellular lipids but no faster in trained than in sedentary subjects. Am. J. Physiol. Regul. Integr. Comp. Physiol. 281:R760-R769.

25. Bogardus, C., et al. 1984. Effects of physical training and diet therapy on carbohydrate metabolism in patients with glucose intolerance and noninsulin-dependent diabetes mellitus. Diabetes. 33:311-318.

26. DeFronzo, R.A., Sherwin, R.S., and Kraemer, N. 1987. Effect of physical training on insulin action in obesity. Diabetes. 36:1379-1385.

27. Richter, E.A., et al. 1989. Effect of exercise on insulin action in human skeletal muscle. J. Appl. Physiol. 66:876-885.

28. Perseghin, G., et al. 1996. Increased glucose transport-phosphorylation and muscle glycogen synthesis after exercise training in insulin-resistant subjects. N. Engl. J. Med. 335:1357-1362.

29. Dela, F., et al. 1995. Insulin-stimulated muscle glucose clearance in patients with NIDDM. Effects of one-legged physical training. Diabetes. 44:1010-1020.

30. Goodpaster, B.H., Katsiaras, A., and Kelley, D.E. 2003. Enhanced fat oxidation through physical activity is associated with improvements in insulin sensitivity in obesity. Diabetes. 52:2191-2197.

31. Short, K.R., et al. 2003. Impact of aerobic exercise training on age-related changes in insulin sensitivity and muscle oxidative capacity. Diabetes. 52:1888-1896.

32. Bruce, C.R., et al. 2006. Endurance training in obese humans improves glucose tolerance and mitochondrial fatty acid oxidation and alters muscle lipid content. Am. J. Physiol. Endocrinol. Metab. 291:E99-E107.

33. Russell, A.P. 2004. Lipotoxicity: the obese and endurance-trained paradox. Int. J. Obes. Relat. Metab. Disord. 28(Suppl. 4):S66-S71.

34. Bassel-Duby, R., and Olson, E.N. 2006. Signaling pathways in skeletal muscle remodeling. Annu. Rev. Biochem. 75:19-37.

35. Etgen, G.J., Jr., et al. 1996. Glucose transport and cell surface GLUT-4 protein in skeletal muscle of the obese Zucker rat. Am. J. Physiol. 271:E294-E301.

36. Hickey, M.S., et al. 1995. Skeletal muscle fiber composition is related to adiposity and in vitro glucose transport rate in humans. Am. J. Physiol. 268:E453-E457.

37. Song, X.M., et al. 1999. Muscle fiber type specificity in insulin signal transduction. Am. J. Physiol. 277:R1690-R1696.

38. Henriksen, E.J., et al. 1990. Glucose transporter protein content and glucose transport capacity in rat skeletal muscles. Am. J. Physiol. 259:E593-E598.
39. Ryder, J.W., et al. 2003. Skeletal muscle reprogramming by activation of calcineurin improves insulin action on metabolic pathways. J. Biol. Chem. 278:44298-44304.

40. Bell, R.M., and Coleman, R.A. 1980. Enzymes of glycerolipid synthesis in eukaryotes. Annu. Rev. Biochem. 49:459-487.

41. Lehner, R., and Kuksis, A. 1996. Biosynthesis of triacylglycerols. Prog. Lipid Res. 35:169-201.

42. Bagnato, C., and Igal, R.A. 2003. Overexpression of diacylglycerol acyltransferase- 1 reduces phospholipid synthesis, proliferation, and invasiveness in simian virus 40-transformed human lung fibroblasts. J. Biol. Chem. 278:52203-52211.

43. Oelkers, P., et al. 1998. Characterization of two human genes encoding acyl coenzyme A:cholesterol acyltransferase-related enzymes. J. Biol. Chem. 273:26765-26771.

44. Cases, S., et al. 1998. Identification of a gene encoding an acyl CoA:diacylglycerol acyltransferase, a key enzyme in triacylglycerol synthesis. Proc. Natl. Acad. Sci. U. S. A. 95:13018-13023.

45. Cases, S., et al. 2001. Cloning of dgat2, a second mammalian diacylglycerol acyltransferase, and related family members. J. Biol. Chem. 276:38870-38876.

46. Lardizabal, K.D., et al. 2001. Dgat2 is a new diacylglycerol acyltransferase gene family: purification, cloning, and expression in insect cells of two polypeptides from mortierella ramanniana with diacylglycerol acyltransferase activity. J. Biol. Chem. 276:38862-38869.

47. Meegalla, R.L., Billheimer, J.T., and Cheng, D. 2002. Concerted elevation of acyl-coenzyme A:diacylglycerol acyltransferase (DGAT) activity through independent stimulation of mRNA expression of DGAT1 and DGAT2 by carbohydrate and insulin. Biochem. Biophys. Res. Commun. 298:317-323.

48. Smith, S.J., et al. 2000. Obesity resistance and multiple mechanisms of triglyceride synthesis in mice lacking Dgat. Nat. Genet. 25:87-90.

49. Stone, S.J., et al. 2004. Lipopenia and skin barrier abnormalities in DGAT2-deficient mice. J. Biol. Chem. 279:11767-11776.

50. Yen, C.L., et al. 2005. The triacylglycerol synthesis enzyme DGAT1 also catalyzes the synthesis of diacylglycerols, waxes, and retinyl esters. J. Lipid Res. 46:1502-1511.

51. Orland, M.D., et al. 2005. Acyl coenzyme A dependent retinol esterification by acyl coenzyme A: diacylglycerol acyltransferase 1. Biochim. Biophys. Acta. 1737:76-82.

52. Ikeda, S., et al. 2002. Up-regulation of SREBP$1 \mathrm{c}$ and lipogenic genes in skeletal muscles after exercise training. Biochem. Biophys. Res. Commun. 296:395-400.

53. Avignon, A., et al. 1996. Chronic activation of protein kinase $\mathrm{C}$ in soleus muscles and other tissues of insulin-resistant type II diabetic Goto-Kakizaki (GK), obese/aged, and obese/Zucker rats. A mechanism for inhibiting glycogen synthesis. Diabetes. 45:1396-1404.

54. Schmitz-Peiffer, C., et al. 1997. Alterations in the expression and cellular localization of protein kinase $\mathrm{C}$ isozymes epsilon and theta are associated with insulin resistance in skeletal muscle of the high-fat-fed rat. Diabetes. 46:169-178.

55. Itani, S.I., et al. 2000. Involvement of protein kinase $\mathrm{C}$ in human skeletal muscle insulin resistance and 
obesity. Diabetes. 49:1353-1358.

56. Itani, S.I., et al. 2002. Lipid-induced insulin resistance in human muscle is associated with changes in diacylglycerol, protein kinase C, and IkappaBalpha. Diabetes. 51:2005-2011.

57. Griffin, M.E., et al. 1999. Free fatty acid-induced insulin resistance is associated with activation of protein kinase $\mathrm{C}$ theta and alterations in the insulin signaling cascade. Diabetes. 48:1270-1274.

58. Kim, J.K., et al. 2004. PKC-theta knockout mice are protected from fat-induced insulin resistance. J. Clin. Invest. 114:823-827. doi:10.1172/ JCI200422230.

59. Farese, R.V., Sajan, M.P., and Standaert, M.L. 2005. Atypical protein kinase $\mathrm{C}$ in insulin action and insulin resistance. Biochem. Soc. Trans. 33:350-353.

60. Kaneki, M., et al. 1999. Functional role for protein kinase Cbeta as a regulator of stress-activated protein kinase activation and monocytic differentiation of myeloid leukemia cells. Mol. Cell. Biol. 19:461-470.

61. Werlen, G., et al. 1998. Calcineurin preferentially synergizes with PKC-theta to activate JNK and IL-2 promoter in T lymphocytes. EMBO J. 17:3101-3111.

62. Aguirre, V., et al. 2000. The c-Jun NH(2)-terminal kinase promotes insulin resistance during association with insulin receptor substrate- 1 and phosphorylation of Ser(307). J. Biol. Chem. 275:9047-9054.

63. Aguirre, V., et al. 2002. Phosphorylation of Ser307 in insulin receptor substrate-1 blocks interactions with the insulin receptor and inhibits insulin action. J. Biol. Chem. 277:1531-1537.

64. Trushin, S.A., et al. 1999. Protein kinase C and calcineurin synergize to activate IkappaB kinase and NF-kappaB in T lymphocytes. J. Biol. Chem.
274:22923-22931.

65. Gao, Z., et al. 2002. Serine phosphorylation of insulin receptor substrate 1 by inhibitor kappa B kinase complex. J. Biol. Chem. 277:48115-48121.

66. Chen, H.C., et al. 2002. Analysis of energy expenditure at different ambient temperatures in mice lacking DGAT1. Am. J. Physiol. Endocrinol. Metab. 284:E213-E218.

67. Chen, H.C., et al. 2002. Increased insulin and leptin sensitivity in mice lacking acyl CoA:diacylglycerol acyltransferase 1. J. Clin. Invest. 109:1049-1055. doi:10.1172/JCI200214672.

68. Chen, H.C., et al. 2004. Role of adipocyte-derived factors in enhancing insulin signaling in skeletal muscle and white adipose tissue of mice lacking Acyl CoA:diacylglycerol acyltransferase 1. Diabetes. 53:1445-1451.

69. Chen, H.C., et al. 2003. Obesity resistance and enhanced glucose metabolism in mice transplanted with white adipose tissue lacking acyl CoA:diacylglycerol acyltransferase 1. J. Clin. Invest. 111:1715-1722. doi:10.1172/JCI200315859.

70. Listenberger, L.L., et al. 2003. Triglyceride accumulation protects against fatty acid-induced lipotoxicity. Proc. Natl. Acad. Sci. U. S. A. 100:3077-3082.

71. Haemmerle, G., et al. 2006. Defective lipolysis and altered energy metabolism in mice lacking adipose triglyceride lipase. Science. 312:734-737.

72. Serra, C., et al. 2003. Transgenic mice with dominant negative PKC-theta in skeletal muscle: a new model of insulin resistance and obesity. J. Cell. Physiol. 196:89-97.

73. Gao, Z., et al. 2007. Inactivation of PKCtheta leads to increased susceptibility to obesity and dietary insulin resistance in mice. Am. J. Physiol. Endocrinol.
Metab. 292:E84-E91.

74. Bronfman, M., Morales, M.N., and Orellana, A. 1988. Diacylglycerol activation of protein kinase $\mathrm{C}$ is modulated by long-chain acyl-CoA. Biochem. Biophys. Res. Commun. 152:987-992.

75. Summers, S.A., et al. 1998. Regulation of insulinstimulated glucose transporter GLUT4 translocation and Akt kinase activity by ceramide. Mol. Cell. Biol. 18:5457-5464.

76. Schmitz-Peiffer, C., Craig, D.L., and Biden, T.J. 1999. Ceramide generation is sufficient to account for the inhibition of the insulin-stimulated PKB pathway in C2C12 skeletal muscle cells pretreated with palmitate. J. Biol. Chem. 274:24202-24210.

77. Couplan, E., et al. 2002. High level of uncoupling protein 1 expression in muscle of transgenic mice selectively affects muscles at rest and decreases their IIb fiber content. J. Biol. Chem. 277:43079-43088.

78. Yu, Y.H., et al. 2002. Posttranscriptional control of the expression and function of diacylglycerol acyltransferase- 1 in mouse adipocytes. J. Biol. Chem. 277:50876-50884.

79. Chen, N., et al. 2005. Whole-body insulin resistance in the absence of obesity in FVB mice with overexpression of Dgat 1 in adipose tissue. Diabetes. 54:3379-3386.

80. Blaner, W.S. 1990. Radioimmunoassays for retinolbinding protein, cellular retinol-binding protein, and cellular retinoic acid-binding protein. Methods Enzymol. 189:270-281.

81. Liu, L., et al. 2001. Nuclear factor kappa B is involved in lipopolysaccharide-stimulated induction of interferon regulatory factor- 1 and GAS/ GAF DNA-binding in human umbilical vein endothelial cells. Br. J. Pharmacol. 134:1629-1638. 